\title{
Exodontias múltiplas associadas à alveoloplastia com finalidade de reabilitação protética: relato de caso clínico
}

\author{
Multiple exodonties associated with alveoloplasty for the prosthetic rehabilitation: clinical case
} report

Exodoncias múltiples asociadas con alveoloplastia para la rehabilitación protésica: reporte de caso clínico

\author{
Isabela Soniely Maria da Silva França \\ ORCID: https://orcid.org/0000-0002-7060-3355 \\ Centro Universitário Brasileiro, Brasil \\ E-mail: isabela_silva.com@hotmail.com \\ Maria Emanuella Letícia da Silva \\ ORCID: https://orcid.org/0000-0002-7159-1165 \\ Centro Universitário Brasileiro, Brasil \\ E-mail: emanuelaleticia@hotmail.com \\ Luís Felipe Espíndola-Castro \\ ORCID: https://orcid.org/0000-0002-1923-8057 \\ Universidade de Pernambuco, Brasil \\ E-mail: drfelipeespindola@gmail.com \\ Nathalia Gomes de Oliveira \\ ORCID: https://orcid.org/0000-0001-6937-1537 \\ Universidade de Pernambuco, Brasil \\ E-mail: nataliagomes04@hotmail.com \\ Emerson Filipe de Carvalho Nogueira \\ ORCID: https://orcid.org/0000-0002-4560-7733 \\ Universidade de Pernambuco, Brasil \\ E-mail: emerson_filipe@ hotmail.com \\ Renata de Albuquerque Cavalcanti Almeida \\ ORCID: https://orcid.org/0000-0003-1101-3491 \\ Universidade de Pernambuco, Brasil \\ E-mail: realmeida81@outlook.com
}

\section{Resumo}

Introdução: Existem diversas razões associadas à perda dentária que podem desencadear na necessidade de realização de exodontias múltiplas, apresentando comprovada eficácia na eliminação de focos infecciosos e na manutenção da saúde bucal e geral do indivíduo. Ao perder os dentes, o paciente modifica seu padrão de fala, estética, mastigação e deglutição, resultando em restrições funcionais, sociais e psicológicas. Alveoloplastia é um procedimento cirúrgico que possibilita a correção plástica do processo alveolar, objetivando uniformizar o tecido ósseo remanescente, permitindo melhor adaptação protética necessária após as exodontias. Objetivo: relatar um caso clínico de exodontias múltiplas associadas à alveoloplastia com finalidade protética e mostrar a importância de um planejamento adequado. Relato de caso: Paciente procurou a clínica odontológica de uma instituição de ensino privada, queixando-se de dor nos dentes ao se alimentar e falta de estética ao sorrir. Ao exame clínico, observou-se presença de restos radiculares e dentes com mobilidades. $\mathrm{O}$ tratamento proposto foi realização de múltiplas exodontias associadas à cirurgia para regularização do rebordo alveolar e posteriormente encaminhamento para reabilitação protética. As próteses dentárias total mucossuportada superior e parcial dentomucossuportada inferior foram indicadas para o caso relatado mediante a necessidade do paciente, sua condição financeira e a possibilidade de tratamento a ser realizado na instituição onde foi realizado o tratamento. Conclusão: A reabilitação com próteses dentárias possui vantagens, tanto no âmbito funcional, normalizando a fonética, e principalmente, desenvolvendo a função mastigatória, como no âmbito social, reestabelecendo a estética, devolvendo o sorriso e a auto-estima do indivíduo.

Palavras-chave: Cirurgia bucal; Extração dentária; Alveoloplastia; Prótese dentária.

\section{Abstract}

Introduction: There are several reasons associated with tooth loss that can trigger the need for multiple extractions, showing proven efficacy in the elimination of infectious foci and in maintaining the individual's oral and general health. Upon losing teeth, the patient changes his speech, aesthetics, chewing and swallowing pattern, resulting in 
functional, social and psychological restrictions. Alveoloplasty is a surgical procedure that allows the plastic correction of the alveolar process, aiming to standardize the remaining bone tissue, allowing for the best prosthetic adaptation necessary after extractions. Objective: to report a clinical case of multiple extractions associated with alveoloplasty for prosthetic purposes and to show the importance of proper planning. Case report: The patient sought the dental clinic of a private educational institution, complaining of pain in the teeth when eating and lack of aesthetics when smiling. Clinical examination revealed the presence of root debris and teeth with mobility. The proposed treatment was to perform multiple extractions associated with surgery to regularize the alveolar ridge and later forward to prosthetic rehabilitation. The total upper mucosal supported dental prostheses and partial lower dentomucosal supported dentures were indicated for the case reported according to the patient's need, financial condition and the possibility of treatment to be performed at the institution where the treatment was performed. Conclusion: Rehabilitation with dental prostheses has advantages, both in the functional realm, normalizing phonetics, and mainly, developing the masticatory function, as well as in the social realm, restoring aesthetics, restoring the individual's smile and self-esteem.

Keywords: Oral surgery; Dental extraction; Alveoloplasty; Dental prosthesis.

\section{Resumen}

Introducción: Son varios los motivos asociados a la pérdida dentaria que pueden desencadenar la necesidad de múltiples extracciones, demostrando eficacia probada en la eliminación de focos infecciosos y en el mantenimiento de la salud bucal y general del individuo. Al perder los dientes, el paciente cambia el habla, la estética, el patrón de masticación y deglución, lo que genera restricciones funcionales, sociales y psicológicas. La alveoloplastia es un procedimiento quirúrgico que permite la corrección plástica del proceso alveolar, con el objetivo de estandarizar el tejido óseo remanente, permitiendo la mejor adaptación protésica necesaria tras las extracciones. Objetivo: reportar un caso clínico de extracciones múltiples asociadas a alveoloplastia con fines protésicos y mostrar la importancia de una adecuada planificación. Caso clínico: El paciente acudió a la clínica dental de una institución educativa privada, quejándose de dolor en los dientes al comer y falta de estética al sonreír. El examen clínico reveló la presencia de restos radiculares y dientes con movilidad. El tratamiento propuesto fue realizar múltiples extracciones asociadas a la cirugía para regularizar el reborde alveolar y posteriormente adelantar a la rehabilitación protésica. Se indicaron las prótesis dentales totales con soporte mucoso superior y las prótesis parcial con soporte dentomucoso inferior para el caso reportado de acuerdo con la necesidad del paciente, condición económica y posibilidad de tratamiento a realizar en la institución donde se realizó el tratamiento. Conclusión: La rehabilitación con prótesis dentales tiene ventajas, tanto en el ámbito funcional, normalizando la fonética, y principalmente, desarrollando la función masticatoria, como en el ámbito social, restableciendo la estética, restaurando la sonrisa y la autoestima del individuo.

Palabras clave: Cirugía bucal; Extracción dental; Alveoloplastia; Prótesis dental.

\section{Introdução}

Segundo HUPP et al., (2009), exodontias múltiplas são sequências de cirurgias pela técnica aberta em uma mesma sessão. Pacientes com a dentição prejudicada, possuem características únicas que desafiam o cirurgião-dentista quanto ao reestabelecimento das funções do sistema estomatognático. Neste contexto, uma vez diagnosticado clínica e radiograficamente a necessidade de múltiplas extrações dentárias, uma forma de melhorar a qualidade de vida do paciente é reabilitando-o. Esse tipo de procedimento torna-se essencial em alguns casos, devido a necessidade da eliminação do foco infeccioso e manutenção da saúde bucal e geral do paciente (Seco et al., 2019).

Existem várias situações em que exodontias múltiplas são indicadas, como grande comprometimento dental por cáries extensas em dentes próximos, necrose pulpar, doenças periodontais graves, razões estéticas, dentes fraturados, motivos protéticos, mobilidade e ausência de procedimento conservador viável (Miraglia et al., 2000; Gonçalves et al., 2020).

As técnicas cirúrgicas pré-protéticas podem melhorar as condições anatômicas de rebordos e vestíbulos pós exodontias, facilitando a instalação das próteses removíveis. As cirurgias pré-protéticas podem ser realizadas em tecidos duros ou em tecidos moles. As abordagens pré-protéticas em tecido duro englobam: exodontias, alveoloplastias, remoção ou regularização do tubérculo geniano, exostoses, tórus mandibular e palatino e crista milohióidea (Soares et al., 2020; Cardoso et al., 2016).

Alveoloplastia é um procedimento cirúrgico que possibilita a correção plástica do processo alveolar com o objetivo de uniformizar o tecido ósseo remanescente, permitindo uma melhor adaptação da prótese a ser colocada após as extrações, deixando o paciente apto para receber a prótese (Hanna et al., 2011). 
Para a reabilitação de pacientes que sofreram grandes perdas dentárias como no caso de exodontias múltiplas, deve-se sempre buscar o restabelecimento da função e estética do paciente. Para isto existem diversas possibilidades: prótese fixa sobre implante ou móvel sobre implantes (overdenture), prótese dentomucossuportada (parcial removível) ou próteses totais mucossuportadas. A indicação de cada uma das alternativas de reabilitação depende da condição financeira do paciente, da perda óssea e dentária envolvida, e de algum tipo de doença de base que o paciente apresente que contraindique um procedimento cirúrgico. O plano de tratamento deve ser individualizado e sempre ouvindo as expectativas do paciente, com o intuito de atender suas necessidades. (Marchini et al., 2010; Sugio et al., 2019).

A cirurgia pré-protética é, pois, parte integrante da cirurgia oral e maxilofacial e da prótese dentária. O desenvolvimento de um planejamento adequado é o momento crucial para definir o prognostico do tratamento e consequentemente do paciente, portanto, deve envolver a interrelação entre o protesista e o cirurgião bucomaxilofacial (Aggarwal; Cho, 2014; Kolokythas; Jamali; Miloro, 2015). O presente estudo tem por objetivo relatar um caso clínico e mostrar a importância de se planejar de forma multidisciplinas os \tratamentos, visando à reabilitação do paciente dentro de sua realidade.

\section{Relato de Caso}

Paciente J.A.G.S, sexo masculino, 45 anos, leucoderma, procurou a Clínica Odontológica de uma instituição de ensino superior, queixando-se de dor nos dentes ao se alimentar e falta de estética ao sorrir. Na anamnese, paciente relata não apresentar nenhum comprometimento sistêmico ou alergia.

No exame clínico, todos os dentes superiores existentes encontravam-se comprometidos, com presença de restos radiculares dos dentes 11, 12, 13, 21, 22, 23 e dentes 26 e 16 cariados, com mobilidade grau II, exposição do terço cervical das raízes palatinas. Na arcada inferior, havia restos radiculares dos dentes 44, 48 e 34, além da presença dos dentes 41 , 42 , que apresentavam-se com certa mobilidade e dos hígidos 31, 32, 33 e 43. (Figura 1).

Figura 1. Aspecto clínico inicial.

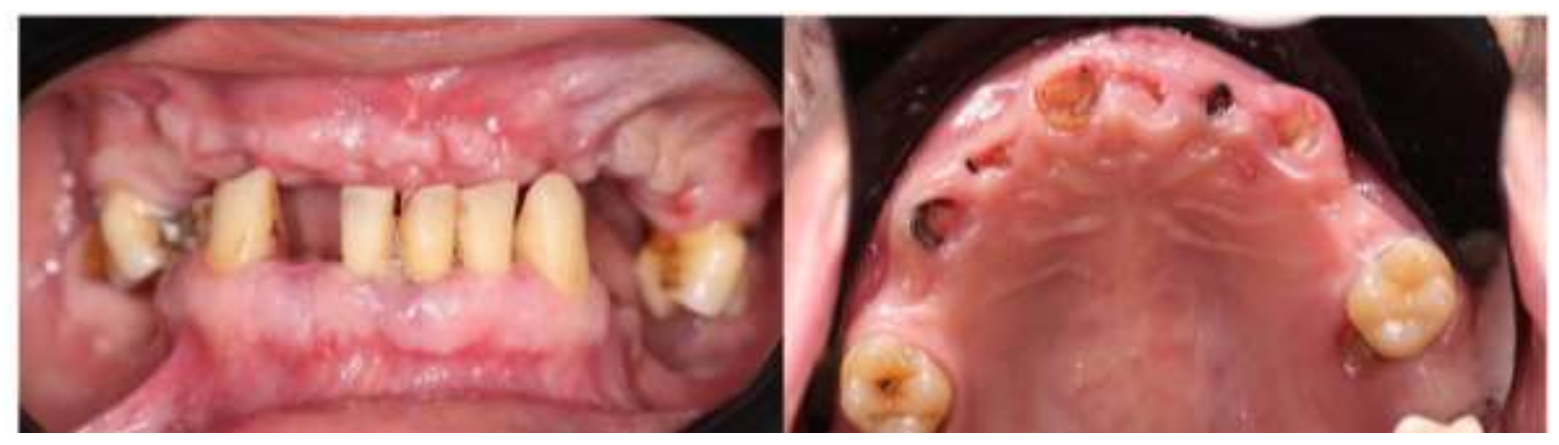

Fonte: Autores.

O paciente foi informado sobre a importância da manutenção da saúde oral e da necessidade de remoção de todos os elementos dentários comprometidos, onde foi solicitado radiografia panorâmica dos maxilares para adequado planejamento (Figura 2). 
Figura 2. Radiografia panorâmica dos maxilares.

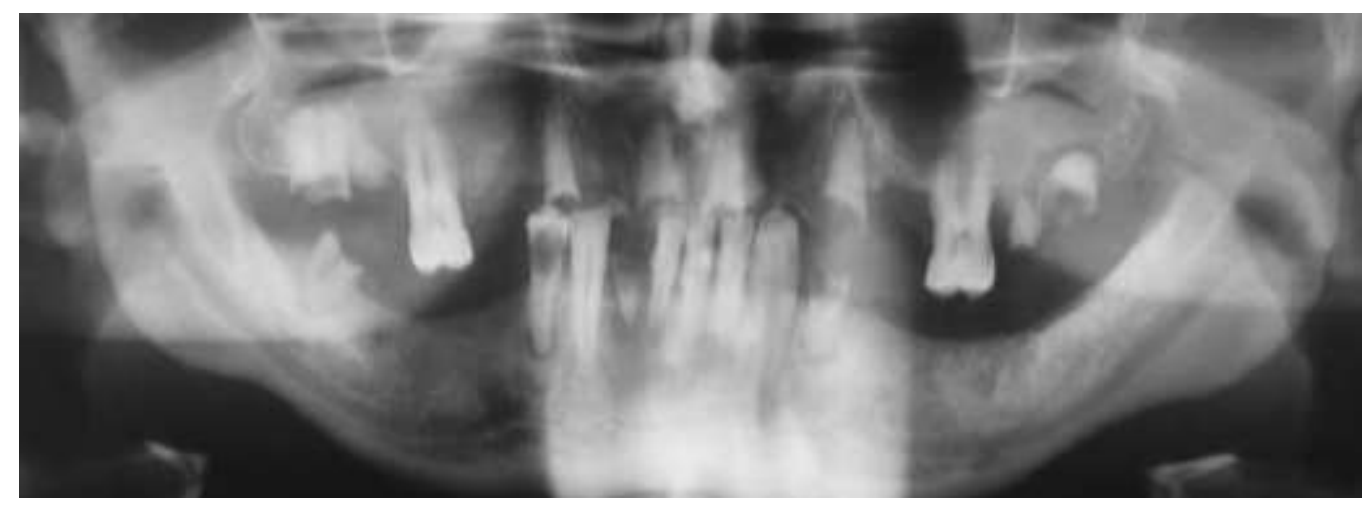

Fonte: Autores.

Com isso, o tratamento de escolha foi a realização de exodontias múltiplas dos restos radiculares da maxila e da mandíbula, mantendo os demais dentes não comprometidos como pilares de sustentação protética e posteriormente reabilitação protética bimaxilar.

As exodontias foram planejadas em dois momentos: exodontias dos dentes indicados da arcada superior e, depois, da inferior. Uma hora antes da cirurgia, foi administrado Dexametasona $8 \mathrm{mg}$, de forma preemptiva, a fim de minimizar a dor, o edema e o trismo pós-operatórios. O procedimento cirúrgico foi realizado sob anestesia local, utilizando-se como anestésico local o cloridrato de mepivacaína a 2\% com adrenalina 1:100.000 (Nova DFL, São Paulo, Brasil). Realizou-se confecção retalho de Newman modificado com descolamento mucoperiosteal (Figura 3), prosseguindo com osteotomia da tábua óssea vestibular com auxílio da caneta de alta rotação e broca cirúrgica nº 702 (broca Carbide FG 702) sob irrigação constante com soro fisiológico $0,9 \%$, com objetivo de expor todos os remanescentes dentários, facilitando sua exodontia.

Figura 3. Retalho de Newmann com descolamento mucoperiosteral.

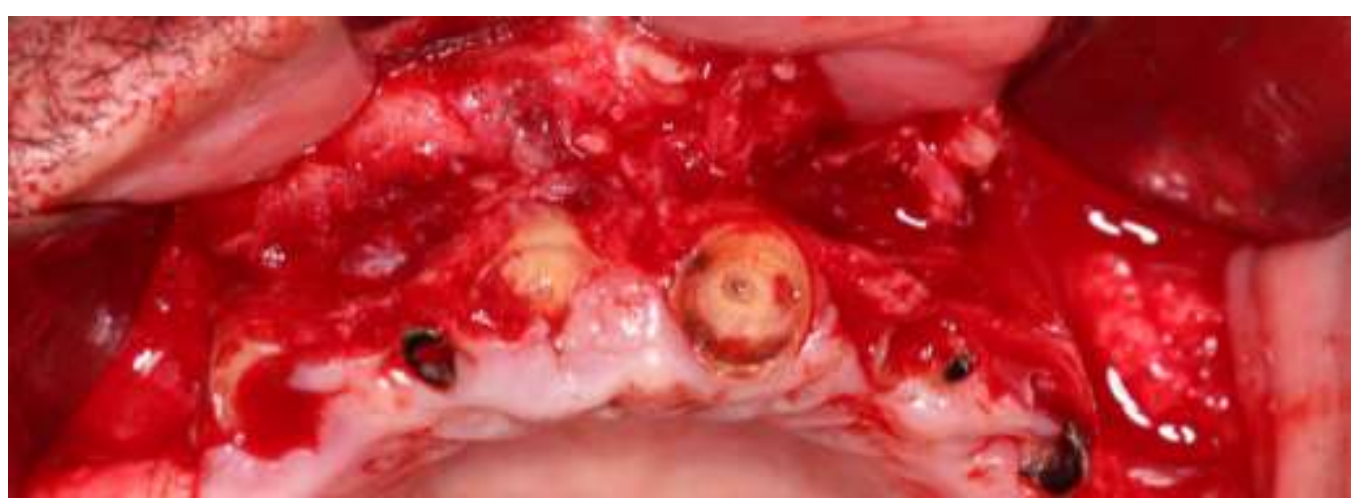

Fonte: Autores.

Após as exodontias, ainda na mesma sessão, foi necessária a realização de alveoloplatia associada, com o objetivo de regularização e remoção de espículas ósseas do rebordo alveolar (Figura 4). 
Figura 4. Regularização do rebordo alveolar.

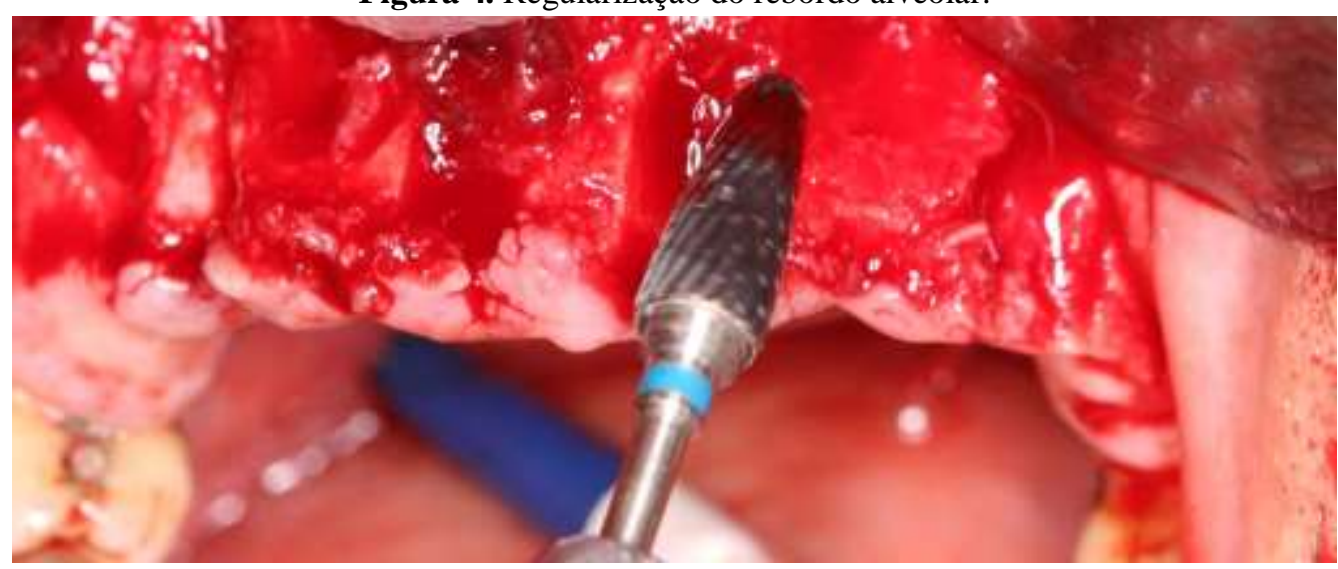

Fonte: Autores.

Para o procedimento, utilizou-se pinça goiva e lima para osso, além de broca Max Cut, promovendo a regularização. A sutura foi realizada com fio de nylon 4-0 (Ethicon - Johnson \& Johnson) e removida após 7 dias (Figura 5).

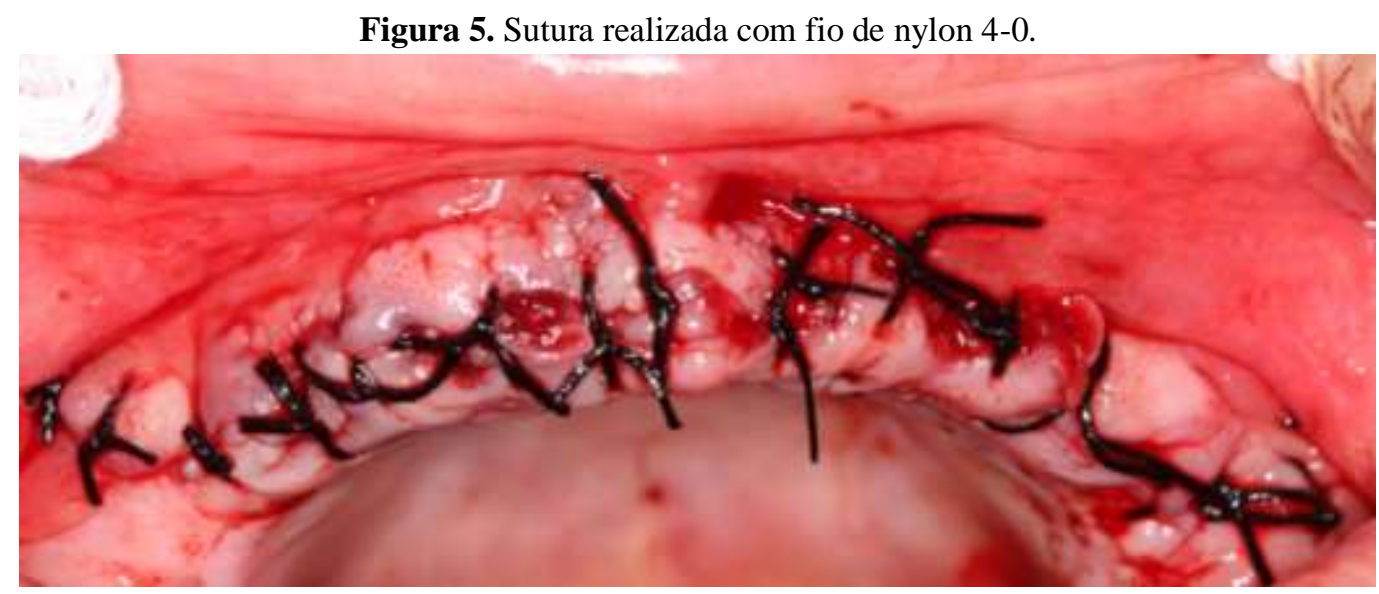

Fonte: Autores.

Para o pós operatório, foi prescrito Ibuprofeno $600 \mathrm{mg}$ de $6 / 6$ horas durante 3 dias), Amoxicilina 500mg de 8/8h por 7 dias, Dipirona Sódica 500mg de 6/6 hrs por 2 dias se caso dor, além do bochecho com digluconato de clorexidina $0,12 \%$, após 24 horas da cirurgia. O paciente evoluiu bem e sem intercorrências após os procedimentos cirúrgicos. Após o período de cicatrização (Figura 6A), foi planejada e realizada a reabilitação protética do paciente, com confecção de prótese total superior mucossuportada e prótese parcial inferior dentomucossuportada (Figura 6B).

Figura 6. A- Rebordo alveolar cicatrizado; B- Paciente reabilitado.

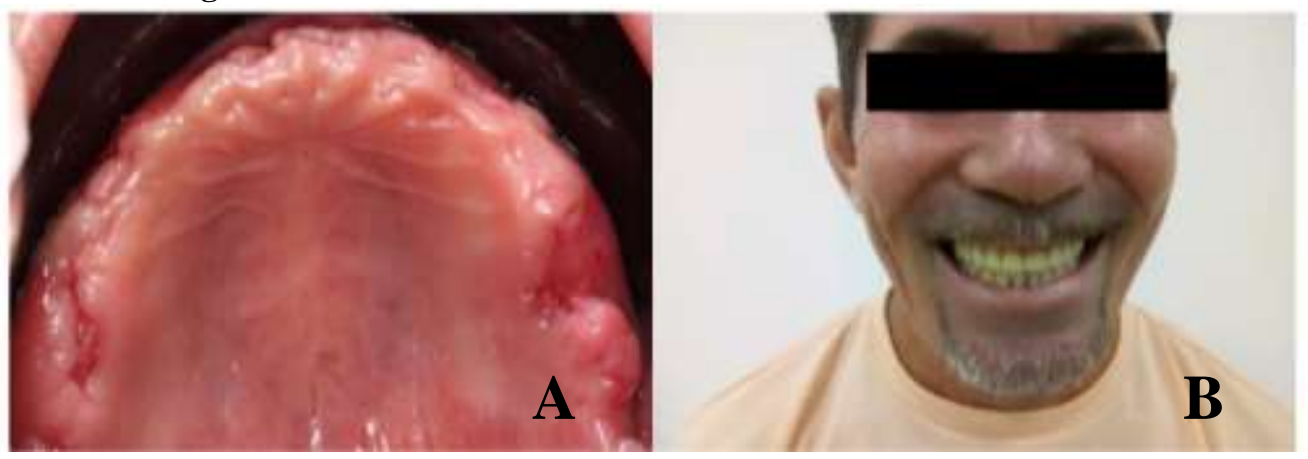

Fonte: Autores. 


\section{Discussão}

Segundo HUPP et al., 2009, um dos principais motivos que acarretam a perda dentária está associada a falta de manutenção da saúde oral, juntamente com a falta de informação sobre a importância desta. Para Miraglia et al., 2000; Gonçalves et al., 2020 as principais indicações de exodontias são: grande comprometimento dental por cáries extensas em dentes próximos, necrose pulpar, doenças periodontais graves, razões estéticas, dentes fraturados, motivos protéticos, mobilidade e ausência de procedimento conservador viável. O paciente em questão, apresentava saúde oral comprometida, com presença diversos restos radiculares além de dentes acometidos por doença periodontal.

Azenha et al., (2012) afirma que a realização das exodontias múltiplas promove a eliminação do foco infeccioso e o restabelecimento da saúde bucal e geral do paciente tendo em vista um tratamento multidisciplinar: periodontal, cirúrgico e protético. O planejamento das exodontia múltiplas tanto em maxila como em mandíbula segundo Kolokythas; Jamali; Miloro (2015), correspondem às exodontias estrategicamente realizadas, o que resulta numa transição suave do estado dentado para o edêntulo o que favoreceu melhoria na condição bucal do paciente em questão, possibilitando-o a uma reabilitação protética.

Segundo Fontes et al., (2018) a técnica de retalho Neumann modificado proporciona exposição adequada da área a ser operada, onde há uma melhor visualização, evitando possíveis complicações. A técnica cirúrgica aberta com amplo retalho mucoperiosteal, como retalho de Neumann modificado foi proposto para o procedimento cirúrgico realizado no paciente, onde proporcionou adequada visualização e exposição da área cirúrgica, para realização das exodontias indicadas, assim como a regularização óssea da região operada. A técnica utilizada foi de crucial para obtenção de uma cirurgia eficaz, pois além da visualização, possibilitou a regularização e correção óssea, prevenindo possíveis complicações cirúrgicas.

Os pacientes que são submetidos à exodontia, principalmente quando múltiplas, podem apresentar espículas ou protuberâncias ósseas pós-operatórias. Isso pode acarretar sensações dolorosas pós-operatórias, ulcerações e inflamação se não forem removidas, ou ainda dificultar a reabilitação protética. As próteses devem estar suportadas por tecido firme, sem protuberâncias, inserções musculares ou hiperplasias fibroepiteliais que impeçam uma boa estabilidade, pois, a má adaptação em conjunto com a falta de orientação ao paciente, pode afetar de forma adversa o prognóstico final do tratamento (Hanna et al., 2011).

A falta de atenção à necessidade de regularização óssea após exodontias termina fazendo necessário um novo procedimento cirúrgico antes da reabilitação do paciente, visando essa regularização. Portanto, as exodontias múltiplas associada a uma cuidadosa alveoloplastia deve ser sempre planejada. Cardoso et al., (2016) afirma que, além de direcionar a reabsorção óssea, possibilita a reabilitação imediata, favorece a cicatrização, evita a necessidade de um segundo ato operatório, e traz agilidade no tratamento do paciente.

As exodontias múltiplas além de essencial para eliminação do foco infeccioso, em conjunto com a realização de plastia óssea possibilitou a correta preparação de um rebordo regular para a reabilitação protética do paciente. Corroborando com a literatura, o tratamento de escolha foi uma excelente opção para o caso relatado, sanando as queixas funcionais e estéticas do paciente.

De acordo com Assis et al., (2015), a realização de tratamentos pré-protéticos cirúrgicos e não cirúrgicos são imprescindíveis para o sucesso e longevidade das reabilitações orais por próteses fixas, removíveis e totais. A reabilitação do paciente foi adequada às condições funcionais, estéticas e financeira do paciente, onde foi confeccionou-se uma prótese total superior mucosuportada e uma prótese parcial removível inferior dentomucossuportada. Essa indicação de reabilitação vem para atender às necessidades de condições bucais atuais do paciente, sendo relevante na reabilitação oral do edentulismo, de acordo com Hawerroth et al., (2017). Além disso, Lima et al., (2018) vêem essa forma de reabilitação escolhida para o caso em questão, como uma possibilidade acessível e satisfatória, que possibilita o retorno da função mastigatória, fonética e estética. 
Determinar com clareza as expectativas do paciente na anamnese foi indispensável para se analisar se estas poderiam ser alcançadas. O sucesso do tratamento do caso foi alcançado através do planejamento cirúrgico e protético dentro da realidade do paciente em questão, devolvendo-o, além da função e da estética, a autoestima e o convívio social.

\section{Conclusão}

A reabilitação com próteses dentárias possui vantagens, tanto no âmbito funcional, normalizando a fonética, e principalmente, desenvolvendo a função mastigatória, como no âmbito social, reestabelecendo a estética, devolvendo o sorriso e a auto-estima do indivíduo. Para o sucesso do tratamento, é imprescindível se fazer um planejamento adequado e que seja compatível com a realidade do paciente, visando sempre o tratamento apropriado para cada situação. A alveoloplastia possibilitou a reabilitação protética, sendo um recurso viável e vantajoso, proporcionando ao paciente conforto, estética e reestabelecendo a função do sistema estomatognático.

\section{Referências}

Aggarwal, H., \& Cho, S. H. (2014). Complete removable dental prosthesis with the swing lock system: A clinical report. The Journal of prosthetic dentistry, $112(5), 1035-1037$.

Assis, P. D. D., Figueiredo, D. P., Carvalho, G. T., Vasconcelos, G. T., Vasconcelos, R., Alves, J., \& Cavalvanti, M. T. D. O. et al. (2015). Adequação do meio bucal e realização de tratamentos pré-protéticos para reintegrar o paciente odontológico na sequência de reabilitação oral. Odontologia Clínico-Científica, 14(4), 831-834.

Azenha, M. R., Lacerda, S. A., Bim, A. L., Caliento, R., \& Guiman, S. (2012). Celulite facial de origem odontogênica. Apresentação de 5 casos. Revista de Cirurgia e Traumatologia Buco-maxilo-facial, 12(3), 41-48.

Cardoso, A. C. F. (2016). Cirurgia pré-protética e reabilitação oral com prótese total (Tese de mestrado). Instituto Superior de Ciências da Saúde Egas Moniz, Portugal.

Devaki, V. N., Blau, K., Ramesh, S. B., Arvind, R. J., \& Vekatesan. (2012). Pre-prosthetic surgery: Mandible. Journal of Pharmacy \& Bioallied Sciences, $4(6), 414-416$.

Fontes, F. A., \& Alves, G. S. (2018). Parestesia dos nervos alveolares inferiores e lingual após exodontia de terceiros molares inferiores. Trabalho de Conclusão de Curso (Monografia) - Universidade de Taubaté, São Paulo.

Gonçalves Júnior, U., Fernandes, S. L., Sousa, M. F. S., Boer, N. P., Nóbrega, A. S., \& Arruda, F. J. S. (2020). Carga imedia ta - da exodontia à restauração cerâmica em 30 dias: relato de caso. Journal of Multidisciplinary Dentistry, 10(1), 97-9.

Hanna, L B. (2011). Manobra de Chompret: alterações dimensionais alveolares vestíbulo palatinas em regiões homólogas que foram submetidas a exodontia. Tese de Doutorado, Universidade de São Paulo, SP, Brasil.

Hawerroth, D. (2017). Influência da reabilitação oral na satisfação e na qualidade de vida do desdentado total: revisão de literatura. Odontologia-Pedra Branca, 17-62.

Hupp J, Ellis III E., \& Tucker, M. (2009). Cirurgia Oral e Maxilofacial Contemporânea (5a ed.) Rio de Janeiro, RJ: Elsevier.

Hupp, J. R., Ellis III, E., \& Tucker, M. R. (2015). Cirurgia oral e maxilofacial contemporânea (6a ed.). Elsevier.

Kolokythas, A., Jamali., \& Miloro, M. (2015). Pre-prosthetic Oral Surgery. Manual of Minor Oral Surgery for the General Dentist, 85-312.

Lima, T. M. N. R., Nascimento, A. L. A., Souza, K. G. C. A., Lima, M. J. A., \& Cardoso, M. M. N. (2018). Reabilitação oral por meio de prótese total superior e prótese parcial removível inferior - relato de caso. Archives Of Health Investigation, 7.

Marchini, L., Montenegro, F. L. B., Cunha, V. De Paula. P., \& Santos, J. F. F. (2010). Prótese dentária na Terceira Idade: considerações clínicas e preventivas diversas. Revista Longeviver, 1(1), 2-6.

Miraglia, S. S., Dutra, T., \& Pinto, J. H. N. (2001). Prótese total: análise comparativa da técnica convencional em relação à técnica da zona neutra. Revista da Associação Paulista de Cirurgiões Dentistas, 55(2), 89-93.

Seco, F., Ozelame, A. P., Baldisserotto, S. M., \& Mioso, F. V. (2019). Planejamento cirúrgico-protético na confecção de prótese total imediata: relato de caso clínico. Rev. Odontol. Araçatuba, 40(2), 27-32.

Soares, T. G., Nascimento, F., Costa, M. D. M. A., \& Reis, T. A. (2020). Cirurgias pré-protéticas em tecidos moles e reabilitação de prótese total. Research, Society and Development, $9(11), 6-25$.

Sugio, C. Y. C., Gomes, A. C. G., Marciel, J. G., Procópio, A. L. F., \& Neppelenbroek, K. H. (2019). Considerações sobre os tipos de próteses parciais removíveis e seu impacto na qualidade de vida. Revista Odontológica de Araçatuba, 40(2), 15-21. 